\title{
Medical education needs overhaul to train more user-friendly physicians, AFMC says
}

Published at www.cmaj.ca on Feb. 1

$\mathrm{T}$ he last time Canada's medical education system underwent a comprehensive review, Wilfred Laurier was prime minister and the Ottawa Hockey Club nabbed the Stanley Cup from the Montréal Wanderers. That was in 1910, when Abraham Flexner, an American education expert, wrote a report that transformed medical education in Canada and the United States.

Now, a century later, the Association of Faculties of Medicine of Canada has taken another look at medical education in Canada and, as expressed in a new report, has found that it is again time for change.

"The timeliness of this report cannot be overstated," Dr. Nick Busing, president and chief executive officer of the Association of Faculties of Medicine of Canada, told an audience gathered in Ottawa, Ontario, on Jan. 28 for the report's official launch. A lot has changed in medical practice in recent decades, Busing noted, including a "virtual explosion" of scientific knowledge and incredible advances in medical technology.

In the report, The Future of Medical Education in Canada (FMEC): A Collective Vision for MD Education, the association makes 15 recommendations on how to improve medical education to meet the increasingly complex and diverse needs of Canadians (www.afmc.ca/fmec/pdf/collective _vision.pdf). The recommendations address such things as medical school admissions, the social accountability of physicians and the need to train doctors to be part of interprofessional teams (for a full list, see Box 1).

"Simply put, this report identifies both generally agreed upon and uniquely Canadian challenges in MD education and offers a transformative vision for the way forward," the report

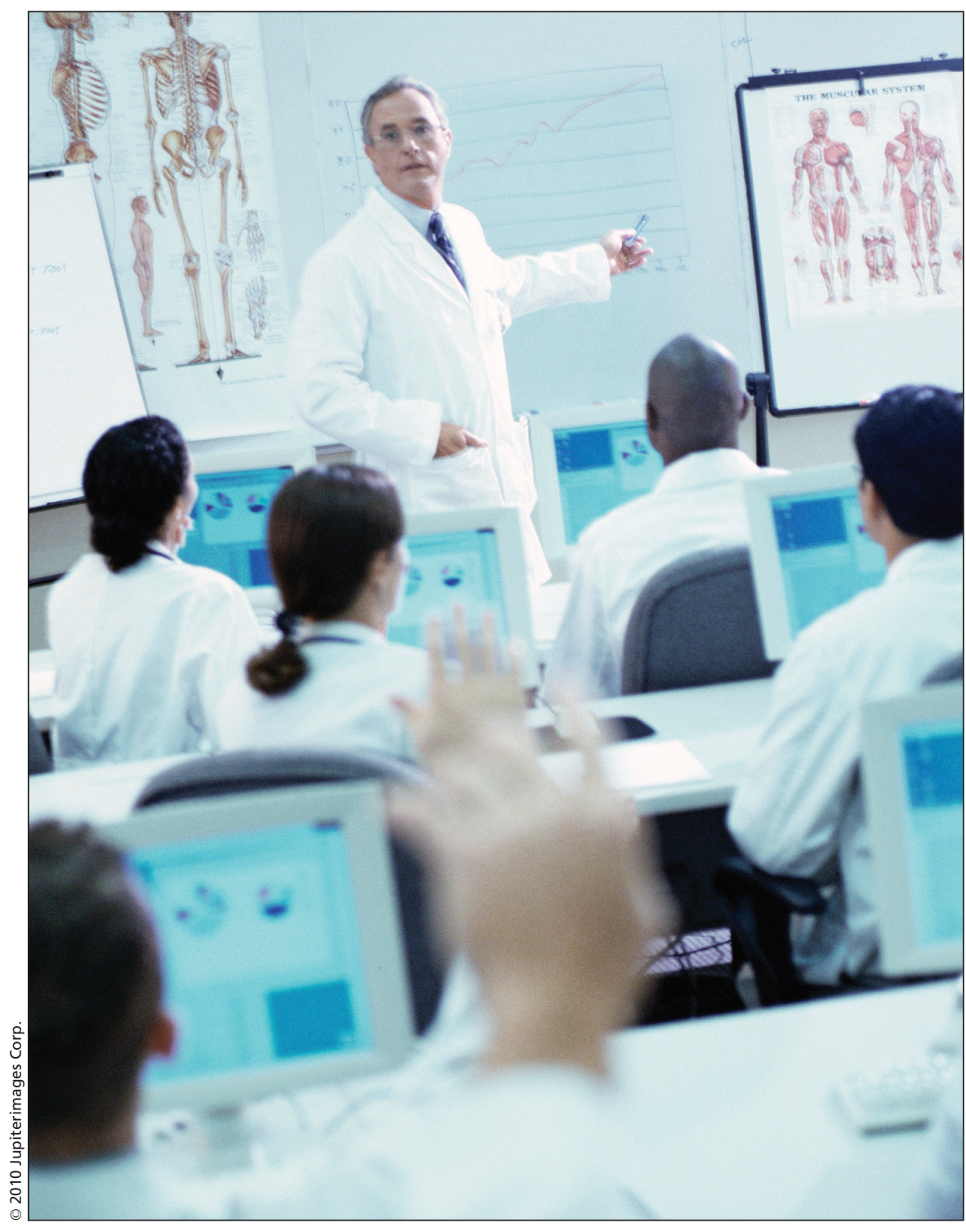

The training of physicians should be extended beyond the medical classroom and teaching hospitals to include community clinic, rural hospitals and other medical facilities, the Association of Faculties of Medicine of Canada says.

states. "It strikes a balance between the impetus for change, what is currently being done, and what remains to be done."

Though the ideas presented in the report aren't new, the FMEC Collective Vision project, which started in 2007, is important because it is a national initia- tive, says Dr. Lewis Tomalty, vicedean of medical education at Queen's University in Kingston, Ontario. Canadian medical schools traditionally focus on improving education in areas most relevant to their regions, he says, but a country-wide vision was needed.

"I think the report has no actual sur- 
prises, but it captures conversations in areas we knew about and put them together," Tomalty says. "What's strong and exciting about this report is that it's taking these things and putting them on paper. It will take a huge amount of work to implement these recommendations and it will not happen overnight, by any stretch, but it gives us something to move forward toward."

At the top of the list of recommendations in the report is to better address community needs. Doctors must be concerned not only with delivering health care services but with being socially responsible and accountable.

"Social accountability is about our need to respond as medical institutions to societal needs in medicine," says Tomalty. "It links directly into things like the fact that we have massive diversity in our population, from rural to urban, from the poor to the affluent."

Another important aspect of medical education that needs to change is the admission process, the report states. Successful candidates for medical school should have more than good grades; their personal values and characteristics - such as communication and interpersonal skills — must also be considered. There is also a need to recruit and support a more diverse mix of students to reflect Canada's diverse population.

"We need to be more inclusive," says Tomalty, who notes that while medical students are more diverse in gender and race than in years past, they still primarily come from the same high socio-economic class. "How do we attract Aboriginal students?"

The report also highlights the need to educate doctors on how to work collaboratively instead of training them as, in Tomalty's words, "isolated entities." There is a move in Canadian health care toward collaborative, patientcentred care, the report states, and medical education should reflect the changes in medical practice, so that new doctors are equipped to work on teams.

The recommendations are the result of more than a year of literature review and analysis, and meetings with Canadian experts on medical education, as well as experts from other countries, such as New Zealand, the United Kingdom and the Netherlands. All 17 Canadian faculties of medicine were consulted on the recommendations after they were drafted.
The Association of Faculties of Medicine of Canada has high hopes for the report, but acknowledges that its effects on transforming medical education in Canada will depend on how medical schools implement the recommendations.

"The FMEC Collective Vision is a prescription for change," the report concludes. "Individual faculties will, no doubt, address each of the recommendations in different ways by establishing priorities in accordance with community needs, faculty strengths, areas needing improvement, and visionary strategic directions for the 21st century. It will be the collective vision and its implementation, however, that truly result in transformative change."

The report follows on the heels of an earlier call by the Association of American Medical Colleges, which represents all 130 US and the 17 accredited Canadian medical schools, to overhaul undergraduate and medical school curricula to focus on bolstering the scientific competency of doctors (CMAJ 2009. DOI:10.1503/cmaj.091197). Roger Collier, CMAJ

DOI:10.1503/cmaj.109-3174

\section{Box I: The Association of Faculties of Medicine's MD education project's full recommendations:}

\section{Recommendations for undergraduate education}

\section{I: Address Individual and Community Needs}

Social responsibility and accountability are core values underpinning the roles of Canadian physicians and Faculties of Medicine. This commitment means that, both individually and collectively, physicians and faculties must respond to the diverse needs of individuals and communities throughout Canada, as well as meet international responsibilities to the global community.

\section{II: Enhance Admissions Processes}

Given the broad range of attitudes, values, and skills required of physicians, Faculties of Medicine must enhance admissions processes to include the assessment of key values and personal characteristics of future physicians-such as communication, interpersonal and collaborative skills, and a range of professional interests-as well as cognitive abilities. In addition, in order to achieve the desired diversity in our physician workforce, Faculties of Medicine must recruit, select, and support a representative mix of medical students.

\section{III: Build on the Scientific Basis of Medicine}

Given that medicine is rooted in fundamental scientific principles, both human and biological sciences must be learned in relevant and immediate clinical contexts throughout the MD education experience. In addition, as scientific inquiry provides the basis for advancing health care, research interests and skills must be developed to foster a new generation of health researchers.

\section{IV: Promote Prevention and Public Health}

Promoting a healthy Canadian population requires a multifaceted approach that engages the full continuum of health and health care. Faculties of Medicine have a critical role to play in enabling this requirement and must therefore enhance the integration of prevention and public health competencies to a greater extent in the MD education curriculum. 


\section{V: Address the Hidden Curriculum}

The hidden curriculum is a "set of influences that function at the level of organizational structure and culture," affecting the nature of learning, professional interactions, and clinical practice. Faculties of Medicine must therefore ensure that the hidden curriculum is regularly identified and addressed by students, educators, and faculty throughout all stages of learning.

\section{VI: Diversify Learning Contexts}

Canadian physicians practise in a wide range of institutional and community settings while providing the continuum of medical care. In order to prepare physicians for these realities, Faculties of Medicine must provide learning experiences throughout MD education for all students in a variety of settings, ranging from small rural communities to complex tertiary health care centres.

\section{VII: Value Generalism}

Recognizing that generalism is foundational for all physicians, MD education must focus on broadly based generalist content, including comprehensive family medicine. Moreover, family physicians and other generalists must be integral participants in all stages of MD education.

\section{VIII: Advance Inter- and Intra-Professional Practice}

To improve collaborative, patient-centred care, MD education must reflect ongoing changes in scopes of practice and health care delivery. Faculties of Medicine must equip MD education learners with the competencies that will enable them to function effectively as part of inter-and intra-professional teams.

IX: Adopt a Competency-Based and Flexible Approach

Physicians must be able to put knowledge, skills, and professional values into practice. Therefore, in this first phase of the medical education continuum, MD education must be based primarily on the development of core foundational competencies and complementary broad experiential learning. In addition to pre-defined curriculum requirements, MD education must provide flexible opportunities for students to pursue individual scholarly interests in medicine.

\section{X: Foster Medical Leadership}

Medical leadership is essential to both patient care and the broader health system. Faculties of Medicine must foster medical leadership in faculty and students, including how to manage, navigate, and help transform medical practice and the health care system in collaboration with others.

\section{Enabling Recommendations to facilitate change in medical education}

\section{A: Realign Accreditation Standards}

Recognizing that accreditation is a powerful lever, Canadian medical leaders must review and realign existing standards of the Committee on Accreditation of Canadian Medical Schools and the Liaison Committee on Medical Education and develop new ones, as necessary, to respond to the recommendations in this report. This may involve the alignment of undergraduate and postgraduate accreditation standards.

\section{B: Build Capacity for Change}

Each Faculty of Medicine should carry out a review of its organizational systems, processes, and structures to determine and build capacity, where required, to support a constructive response to these recommendations.

\section{C: Increase National Collaboration}

Canadian Faculties of Medicine are continually innovating and have much to offer each other. Increased collaboration among schools is needed, including the sharing of teaching and learning resources, evaluation frameworks, tools for common curriculum development, innovations, and information technologies.

\section{D: Improve the Use of Technology}

Based on rapid and evolving technological changes related to the way people communicate and learn, there must be increased understanding and use of technology on the part of both faculty and learners at all MD education sites.

\section{E: Enhance Faculty Development}

Recognizing that teaching, research, and leadership are core roles for physicians, priority must be given to faculty development, support, and recognition in order to enable teachers and learners to respond effectively to the recommendations in this report 